\title{
A solution of High-precision WLAN positioning based on TDOA and PTP
}

\author{
Junjie Zhou ${ }^{1, \mathrm{a}}$, Yue $\mathrm{Ke}^{2}$, Kan $\mathrm{Yu}^{1}$ and Di Tian ${ }^{1}$ \\ ${ }^{1}$ Wenhua College, Huazhong University of Science and Technology, Wuhan, China \\ ${ }^{2}$ School of Computer, Hubei University of Technology, Wuhan, China
}

\begin{abstract}
For indoor WLAN positioning it is difficult to achieve decimeter or centimeter-level precision questions, to IEEE1588v2 (PTP) precision network clock synchronization technology and the arrival time difference principle (TDOA), combined with non line of sight (NLOS) error mitigation techniques ranging research and design a set based on the existing WLAN (IEEE802.11x series of standards) device with high precision real-time location solutions. First, build a high-performance software-defined radio network communications platform, for accurate measurement of the radio signal arrival time and is calculated based on TDOA location. Secondly, the use of high-precision clock IEEE1588v2 standard equipment PTPGrand-2100 as a network clock source, through the integrated application of multi-hop latency compensation and asymmetric compensation algorithm to ensure nanosecond clock synchronization between the various WLAN access points (AP) accuracy(Experiments show that 1 nanosecond time synchronization error is equivalent to 10 to $30 \mathrm{~cm}$ of positioning error). Finally, based NLOS error becomes stability principle, filtered NLOS error and pre-positioned before the data, eliminate the influence of the greatest degree of NLOS error, the time domain error control in wireless ranging $5 \mathrm{~ns}$ or less to achieve decimeter or more accurately estimate the position. The case can be widely used in mobile Internet, logistics management, mineral exploration, health and other needs of the precise location services (LBS) in other industry sectors.
\end{abstract}

Keywords: High-precision positioning; PTP (Precision Time Protocol);Time Difference of Arrival principle; WLAN; Non-line-ranging error

\section{Introduction}

With the advent of the "Internet of Things" era, the wireless location technology as a key technology, has caused the industry wide attention and domestic and international research. The wireless location technology has a very broad application prospects in many areas of military defense, traffic management, disaster relief, environmental hazards, automation workshop and logistics management. The wireless location services have broad market prospects, by 2020 in China the location-based services (LBS) market scale will reach 396.2 billion yuan ${ }^{[1]}$. Vigorously promote the rapid development of optoelectronic information industry, with the needs of social development and economic construction, more and more industries implementation of sophisticated management. The demand for highaccuracy wireless location technology will show rapid growth, particularly for wide-area decimeter accuracy real-time location, or urban areas of real-time meter level accuracy positioning technology, in the next few years.

The level of positioning accuracy and the size of positioning range of the wireless positioning technology has a direct influence on the performance of the positioning system ${ }^{[2]}$. As WLAN popularity, based on
WLAN (IEEE802.11a / b) wireless location technology is the most common, is the lowest-cost way of positioning. However, the existing WLAN positioning system does not meet these requirements emerging decimeter or centimeter-level high-precision positioning applications. The existing WLAN positioning technology is mainly based on signal strength (RSSI) positioning. This kind of technology is greatly influenced by the signal propagation environment. Its general accuracy is not high and the error is usually more than 20 meters $^{[3]}$, which greatly exceeds the needs of the practical application of many high precision positioning. In the field of wireless precise positioning, the current domestic companies are generally used in the most simple RSSI positioning. So in the technology research and development, far behind the foreign counterparts. The Ekahau company of Finland through the acquisition of signal fingerprint way to get the industry's leading WLAN positioning accuracy, but it is only in the ideal situation to improve the positioning accuracy to 5 meters or so. The UK's Ubisense and Germany's Nanotron can provide decimeter or even centimeter level of highprecision wireless positioning solution[4]. But the two company's solution is not based on common WLAN signals, but based on industrial wireless communication

\footnotetext{
a Corresponding author: zjj0718@126.com

Fund Project: Hubei Provincial Department of Education 2015 annual science and technology research projects (No.B2015193); 2014 Hubei Provincial Higher Teaching research project (No. 2014424); 2014 Hubei Provincial Colleges and Universities outstanding young scientific and technological team plans funded project (No.T201431);2014 Wuhan Office of Information Industry wireless city construction of demonstration projects (No.GCJH2014005).
} 
technology, such as UWB (ultra wideband) and Spread Spectrum Chirp (linear frequency modulation spread spectrum), which means that the two companies of the product can not be used together with ordinary wireless terminals. On the other hand, the current commercial WLAN devices or chips do not support the high accuracy of the signal arrival time record.

\section{Comparative Analysis of WLAN positioning method}

\subsection{Overview of WLAN positioning technology}

Wireless local area network (WLAN) based on IEEE802.11 protocol, based on the radio channel as the computer local area network transmission medium. Which is the combination of computer network and wireless communication technology. It uses wireless multiple access channel as the transmission medium, and provides the function of the traditional wired LAN . The WLAN can truly achieve users at any time, anywhere, broadbandInternetaccess ${ }^{[5]}$.Mobile user demands for im mediacy of information and in-place more and more intense, which gives the location-based services WLAN systems provide a broad space for development. WLAN positioning system technologies include GPS / Compass satellite positioning, based TOA / TDOA / AOA triangulation, the signal strength (RSSI) positioning technology $y^{[5]}$. Among them, the signal strength positioning technology mainly includes two kinds of methods, signal strength fingerprint and signal propagation model.

\subsection{Based on time/Angle measurement positioning}

WLAN networking in general will have a lot of high density low power wireless devices, if all wireless devices did not achieve network clock synchronization, distance is also very difficult to achieve using TDOA estimation. Although it is possible by measuring the arrival time (TOA) to estimate the distance, but indoor environment in user space shorter, AP coverage is generally not more than 100 meters, the radio transmission delay can be neglected, and there is a more serious and diffraction and other non-linear propagation diffraction and multipath component pieces of the same user signal is very close in time, the need for improved resolution of the device to distinguish ${ }^{[6]}$. Therefore, accurate TOA and TDOA estimation, needs the help of more advanceddigital signal processing techn iques to achieve. In addition, many obstacles influencing signal transmission, the wireless signal reflection and scattering. Amplitude components of different paths, phase, time of arrival and the angle of incidence varies, so that the received composite signal has a serious distortion in both amplitude and phase. Therefore, the angle of arrival (AOA) is not suitable for indoor wireless positioning.

\subsection{Based on signal strength positioning technology}

Approximate perception, triangulation and scenario analysis are three basic principles to solve the position. Location Based on WLAN signal strength, based on the three principles of the specific positioning technology were the strongest base method, propagation modeling, and location fingerprinting method ${ }^{[7]}$. WLAN network environment based on the principles and architecture positioning system signal strength can refer to Figure 1.

The simplest method is to locate a wireless access terminal for data communication hotspots position, approximately as the estimated location of the wireless terminal, namely the strongest base station method. The precision of this method is limited by the coverage of AP, and it is difficult to pinpoint. The fingerprint positioning is in WLAN network environment under the condition of unchanged, according to different space a location change trend of wireless signal strength consistency rule the same time, based on scenario analysis principle, by matching the measured signals and the fingerprint similarity between to locate the user terminal. Propagation model positioning is based on the received signal strength varies with propagation distance spatial variability law (That is the signal strength path loss propagation) to determine the physical distance between the user terminal and the WLAN hotspot, which use the triangulation and calculation principles completion positioning. Table 1 is a typical example of the WLAN terminal's scan or listener's return to the AP and its related properties. However, based on the signal strength (RSSI) positioning technology is greatly affected by the signal propagation environment, the general accuracy is not high, the error is typically greater than 20 meters, far beyond the need for high precision positioning of many practical applications.

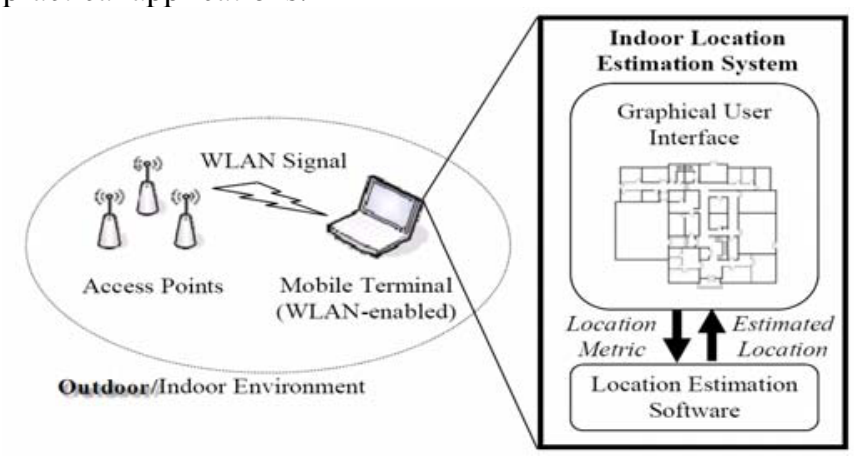

Figure 1. WLAN based on the principle architecture of signal intensity positioning system.

Table 1. AP and its attribute list sample returned by the terminal scan.

\begin{tabular}{|l|l|l|l|}
\hline SSID & MAC & RSSI & Encrypted? \\
\hline Capital City WiFi & $00: 0 f: 34: a b: 0 c: e 0$ & $-66 \mathrm{~dB}$ & No \\
\hline First Security Trust & $00: 0 f: f 7: 0 c: e 9: c 0$ & $-78 \mathrm{~dB}$ & Yes \\
\hline First Security Trust & $00: 0 f: f 7: 0 c: 4 f: 03$ & $-105 \mathrm{~dB}$ & Yes \\
\hline
\end{tabular}




\subsection{WLAN high accuracy positioning scheme b ased on TDOA and PTP}

\subsubsection{The time difference of arrival (TDOA) location technology}

Time difference of arrival (TDOA) positioning, also known as hyperbolic positioning, and is measured by processing the data of three or more measuring stations acquired signals the arrival time measurement data of radiation source to locate ${ }^{[8]}$. In the two-dimensional plane, the radiation source signal arrival time difference between the two measuring station provides a pair of two stations focus hyperbolic. If you use three or four stations to form a two curve, then two of the two pairs of points of intersection, and then use the other auxiliary information to remove the ambiguity, which can determine the location of the radiation source ${ }^{[8]}$. In the three-dimensional space, at least three pairs of doublecurved form to produce an intersection. Hyperbolic form geometric principles is: distance to two fixed points difference locus constant moving point is that two fixed points of hyperbolic focus on two hyperbolic plane intersecting point can be determined.

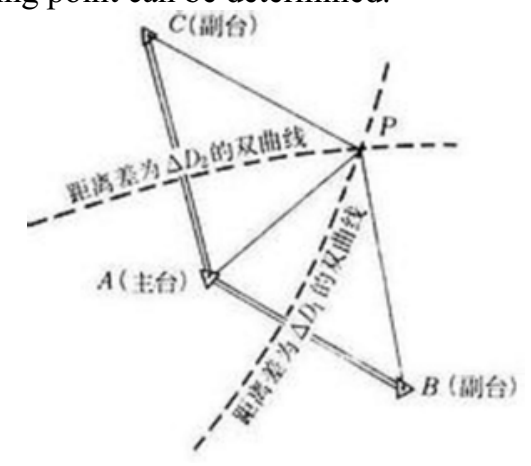

Figure 2. TDOA arrival time difference (hyperbolic) positioning principle

The figure for the position of $\mathrm{P}$ point measured point, A (main station), B (slave station), C (slave station) is 3 reference platform. If the measured distance between $\mathrm{BP}$ and $\mathrm{AP}$ difference $\triangle \mathrm{D} 1, \mathrm{CP}$ and $\mathrm{AP}$ distance difference is $\triangle \mathrm{D} 2$, and $\mathrm{AC}$ distance, $\mathrm{AB}$ distance can be made $\Delta \mathrm{D} 1$, $\triangle \mathrm{D} 2$ draw two hyperbolic on the chart are, the intersection $\mathrm{P}$ is being measuring the position of the point.

TDOA positioning application in WLAN (IEEE $802.11 \mathrm{X}$ ) case is known propagation speed, the radio wave propagation time is proportional to the distance and it spread. But this method needs to pay attention to several problems: (a) radio waves (in the non line of sight NLOS environment) of the transmission characteristics; the general solution is to increase the number of measurements, the measured values obtained statistically significant; (b) clock precision. (c) clock synchronization. We must ensure the synchronization of clocks between participation with a positioning reference point.

Meanwhile, TDOA signal arrival time record with high accuracy requirements, industrial realization is difficult, needs the help of more advanced digital signal processing techniques. Especially WLAN based positioning precision arrival time difference, not only between the wireless access point (AP) to achieve the level of accuracy of time synchronization nanosecond (1 ns time synchronization error corresponding to 10 to 30 $\mathrm{cm}$ of positioning error), further recording error signal arrival time is also needed to control within a few nanoseconds. But now the market is relatively time synchronization accuracy between the only viable AP microsecond or millisecond, and no more mature programs can take advantage of WLAN positioning precision TDOA positioning method. GPS and Compass module can provide nanosecond synchronization accuracy, but wireless AP is usually installed indoors, the position of the blind spot below the satellite signals, so this clock synchronization methods can not meet the requirements of most of the WLAN positioning system.

\subsubsection{IEEE 1588v2 (PTP) precise network clock synchronization technology}

IEEE $1588 \mathrm{v} 2{ }^{[9]}$, with reference to international standards "IEEE Standard for a Precision Clock Synchronization Protocol for Networked Measurement and Control Systems", IEEE Std 1588TM-2008, March 2008. Also known as PTP (Precision Time Protocol), it is a new generation (2008 to complete the agreement for Standardization) clock synchronization technology. Its main feature is applicable to a variety of timing topology, bringing all nodes within a range of IP or Ethernet (such as WLAN AP) synchronized to a common reference clock source (such as GPS / Compass) and achieve submicrosecond (us) synchronization accuracy. PTP clock synchronization network bandwidth is small, reusable AP's existing WLAN network connection. For this reason, we chose to have independent intellectual property rights IEEE1588v2 nanosecond precision clock device PTPGrand-2100 ${ }^{[11]}$, the time source as WLAN network time; and providing PTPWatch network clock monitor, as the network location server and WLAN among TDOA-AP access station and positioning terminal precision clock synchronization monitoring tool when interacting. IEEE1588v2 Master clock device PTP Grand-2100 as shown in Figure 3.

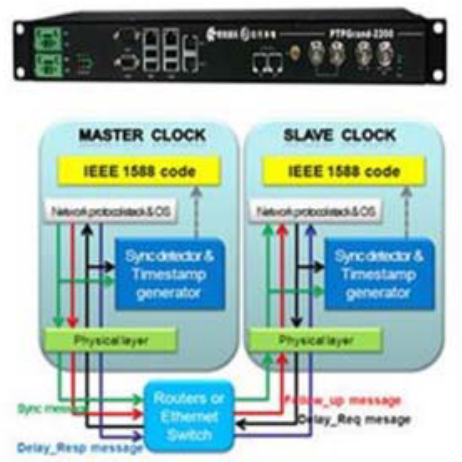

Figure 3. Nanosecond master clock device PTP Grand-2100 and Synchronization software IEEE1588v2 protocol stack.

PTPGrand-2100 embedded IEEE 1588v2 precision network clock protocol stack software (Protocol Stack). The protocol stack to achieve full IEEE 1588v2 functionality, including IEEE1588-2008 protocol engine, 
the best master clock competition (BMC) algorithm, software or hardware time stamp, as well as various clock status and mode of network communication and message standards defined management mechanism ${ }^{[11]}$. The stack either the primary clock, the clock can also be made, depending on the results of BMC algorithm competition or user settings. When an external clock reference source, PTPStack-1000 can be better than $+/$ 50ns (hardware timestamp) or +/- 10us (software timestamp) precision lock the reference source ${ }^{[10]}$. In summary, in order to address indoor WLAN positioning is difficult to achieve decimeter or centimeter precision problem, the research group studied and proposed to reach the time difference principle (TDOA) and IEEE1588v2 (PTP) precise network clock synchronization technology, combined with NLOS ranging error elimination technology, research and design based on a set of high-precision conventional WLAN (IEEE802.11x series of standards) devices (decimeter) RTLS solutions.

\section{The architecture of the high-precision WLAN positioning system}

\subsection{Framework design of the positioning system}

In this design, firstly, we have to TDOA (time difference of arrival) positioning principle as the theoretical basis, construction of a high-performance software-defined radio network communications platform, locating the physical layer to achieve a wireless signal arrival time accurate measurement and signal time difference of arrival (TDOA) calculation. Next, referring to IEEE1588v2 (PTP) international standards, through the integrated application of multi-hop delay compensation and asymmetric compensation algorithm to ensure nanosecond-level time synchronization accuracy (one nanoseconds between WLAN access points (AP) synchronization error equivalent to 10 to $30 \mathrm{~cm}$ positioning error ${ }^{[12]}$ ). Finally, based NLOS error becomes stability principle, through NLOS error filtering and positioning before the data pre-processing, the greatest degree of eliminating the effects of NLOS error, the time domain error control in wireless ranging $5 \mathrm{~ns}$ or less to achieve sub meter level or more accurate location estimate. Accordingly, we propose a design of highprecision positioning system WLAN solutions, as shown in Figure4.

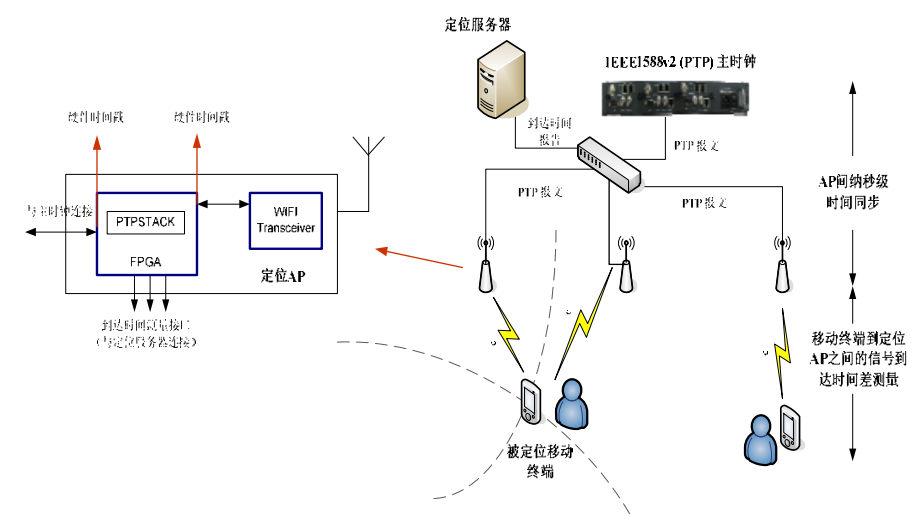

Figure 4.Based on TDOA and the PTP high-precision WLAN positioning system architecture

In the composition of the entire system, including the IEEE1588V2 master clock, TDOA WLAN access station (TDOA-AP), locate the server and the terminal equipment is located. Exploded above figure precision WLAN positioning system, various devices to work together in a unified clock pace to achieve their respective functions.

IEEE1588v2 master clock: using Rubidium timekeeping (GPS optional), by IEEE1588v2 protocol stack and each TDOA WLAN access point (TDOA-AP) interact to synchronize the time between each AP.

TDOA-AP: Each TDOA-AP in addition to traditional wireless network systems, but also added a programmable module (FPGA). FPGA module embedded $1588 \mathrm{v} 2$ precision proprietary module, supports upstream (master clock direction) of the PTP clock synchronization and local clock calibration process packets 1588 hardware timestamp direction from the master clock, and the positioning signals from the mobile terminal direction arrival time stamp, and other functions.

Positioning terminal: Each mobile terminal under the control of the software, the timing to send positioning signals to the AP. Develop positioning terminal to be solved mainly low power consumption, small size and other engineering challenges.

Location server: This server through the network switching device connected to one or more wireless positioning stations, and its main function is to load the system software to summarize the positioning signals provided by TDOA-AP arrival time and the arrival time difference of position calculation and data processing.

\subsection{Key technical analysis and implementation}

To design the high-precision WLAN positioning system based on TDOA and the PTP, the key technical innovations and difficulties mainly reflected in the measurement of high-precision positioning system WLAN physical layer radio signal arrival time, precision clock synchronization between IEEE1588v2 standardsbased AP, data pre-processing NLOS error (NLOS) and the elimination of pre-positioning and other three aspects. Specific solutions and implementation as follows. 


\subsection{1 high-precision measurement of the physical layer signal arrival time}

In order to minimize the error signal arrival time record and improve TDOA location accuracy, we intend through a software-defined radio architecture, a WLAN AP system 802.11MAC, baseband, IF / RF conversion, $\mathrm{RF}$ and power amplifier chip modules together. shown in Figure 5, based on a high-speed programmable gate array (FPGA) and multicore processors DSP development board, through proprietary wireless signal recognition algorithm, implemented on the development board data bus to detect the WiFi signal, stamped. Signal arrival time stamp of playing point (Time-Stamping Point) is in the data path $802.11 \mathrm{M} \mathrm{AC}$ and baseband modules, avoiding the system processing error in the MAC layer and above the protocol stack, thereby positioning arrival signals exact time recording and realtime processing.

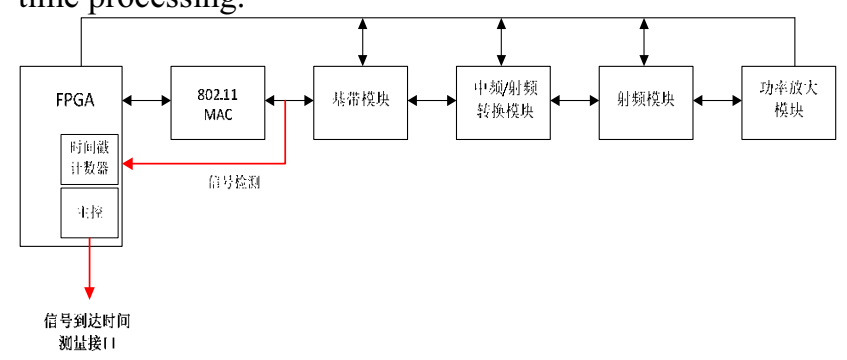

Figure 5. Key Techniques 1: physical layer based on software radio positioning signal measurement time of arrival

\subsubsection{Precision clock synchronization based on IEEE 1588v2 between the AP}

Based on IEEE $1588 \mathrm{v} 2$ precision clock synchronization protocol, we intend to pass a series of technical innovations, including network traffic prediction, multi-hop delay compensation and asymmetric compensation algorithm to achieve the following single-hop 80ns clock synchronization accuracy. This means that the time to use a switch to connect the AP, time recording error AP devices can be controlled in a single measurement $80 \mathrm{~ns}$ or less. As shown in Figure 6, WLAN AP equipment in the work if the load from the master clock, in theory, we calculate the average transmission network information exchange process produces delays. After the adoption of multiple measurements, we will be modeling error distribution (probability distribution to obtain the mean, variance, etc.), the final error control in $5 \mathrm{~ns}$ or less, so that the error can be controlled in TDOA positioning one meter or less.

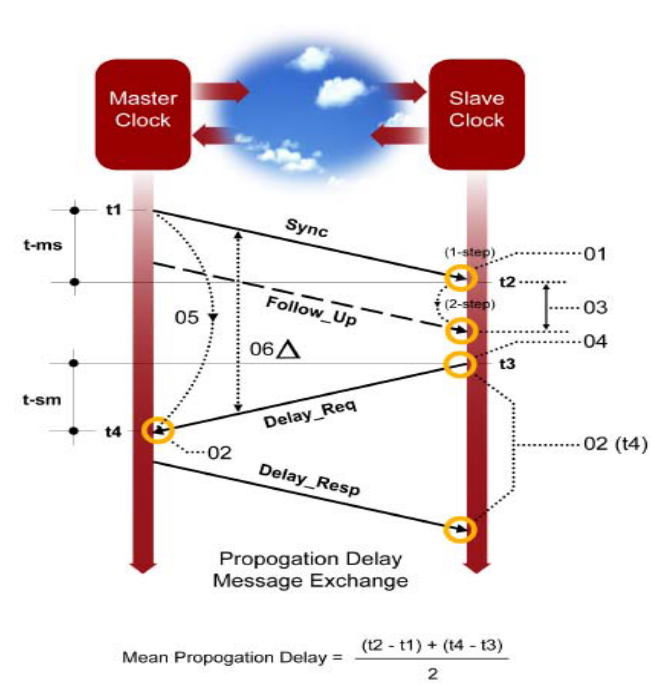

Figure 6. VLAN propagation delay information exchange schematic

\subsubsection{NLOS error cancellation technology}

The presence of a non-line of sight (NLOS) signal transmission between the AP and the positioning signal source is positioned, or ranging TDOA-based location accuracy will be affected. Through theoretical study, using fingerprints and TDOA hybrid positioning signal, and non-line of sight (NLOS) error based on the principle of variable stability NLOS error by filtration and pre-positioned before the data, we can maximize the impact of the elimination of NLOS error achieve decimeter positioning accuracy of less TODA. As shown in Figure 7, based on the principle of variable stability NLOS error when filtered by methods ranging error NLOS geometric analysis ${ }^{[12]}$.

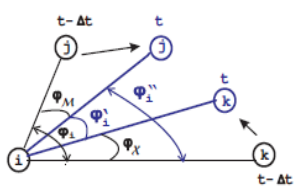

(a)

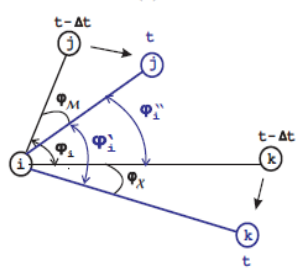

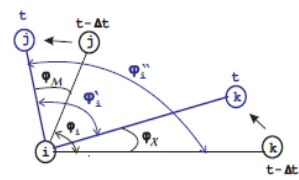

(b)



Figure 7. The NLOS error mitigation Geometric Analysis

In addition, high-precision positioning system WLAN AP also relates to positioning design prototyping, among IEEE1588v2 of TDOA-AP nanoscale clock synchronization algorithm NLOS error filtering algorithms, design and implementation of energy saving algorithms, and embedded hardware and software system integration, commissioning and optimization of the design part. 


\section{Typical applications for solutions and indicators}

With the continuous development of the electronic information technology and the continuous improvement of the degree of social modernization, precise positioning technology, especially based on the precise positioning technology in recent years has been rapid development.In the next few years, there is a need for high precision positioning technology products in the province and the whole country. This kind of positioning technology has already entered the social life and industrial production. The system uses the most common WiFi signal (rather than the other industrial wireless technology), and can be widely for marketing produce huge economic and social benefits. The following table (Table 2) are listed in real time with high precision positioning industry in seven examples of the application and its specific accuracy requirements.

High precision TDOA positioning scheme based on PTP and WLAN is applied to a certain industrial production enterprise, as shown in Figure 8.The positioning system is realized by the method of $10 \mathrm{~cm}$ level accuracy positioning of the human, vehicle and object in a factory. By a finite number of TDOA-AP laid at the factory, real-time accurately positioning workers, forklift trucks, cargo and other micro tag location, people, vehicles and position information recorded in a few nanoseconds to almost zero delay transmit to the control center to a location server. Factory to achieve precise control on lean production and convenient management personnel for regional security control, on-the-job monitoring, vehicle trajectory in real time monitoring.

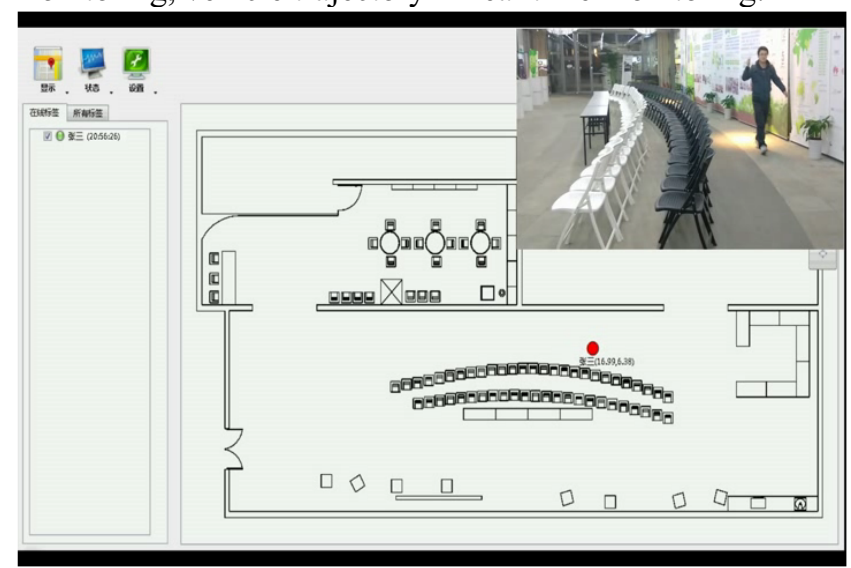

Figure 8. System to achieve precise positioning of the factory workers renderings

This case is a typical application of industrial production of human, vehicle, and material objects such as hybrid location and category management, truly precise, efficient, real-time construction of the Internet of things in the factory. After the completion of the system and repeated testing, the positioning parameters are as follows:

1.WLAN positioning signal: IEEE 802.11X;

2.Time recording error between AP (AP via hop between switches), a single measurement $12 \sim 50 \mathrm{~ns}$, the average of multiple measurements of $25 \mathrm{~ns}$;

3. real-time positioning accuracy: positioning station (TDOA-AP) and positioning the terminal for the next
Line of sight (LOS) conditions mean $9 \mathrm{~cm}$, the positioning station (TDOA-AP) and the positioning terminal is under NLOS conditions mean of $21 \mathrm{~cm}$; 4. positioning terminal standby / charge cycles: $168 \mathrm{~h}$.

Table 2. High-precision positioning Applications in different

\begin{tabular}{|c|c|c|c|}
\hline $\begin{array}{l}\text { Indust } \\
\text { ry }\end{array}$ & $\begin{array}{l}\text { Main } \\
\text { applicatio } \\
\text { ns }\end{array}$ & Application description & $\begin{array}{l}\text { Positi } \\
\text { oning } \\
\text { accura } \\
\text { cy }\end{array}$ \\
\hline $\begin{array}{l}\text { Healthc } \\
\text { are }\end{array}$ & $\begin{array}{l}\text { Medical } \\
\text { equipment } \\
\text { management } \\
\text {; patient } \\
\text { activity } \\
\text { tracking }\end{array}$ & $\begin{array}{l}\text { WLAN positioning in the medical } \\
\text { industry not only can be used to } \\
\text { track the placement of medical } \\
\text { devices but also can be } \\
\text { convenient for medical personnel } \\
\text { to real-time tracking of key } \\
\text { patients }\end{array}$ & $\begin{array}{l}<1 \\
\text { meter } \\
\text { (instru } \\
\text { ment } \\
\text { manag } \\
\text { ement) } \\
<3 \\
\text { meters } \\
\text { (patient } \\
\text { trackin } \\
\text { g) }\end{array}$ \\
\hline $\begin{array}{l}\text { Mining } \\
\text { explorat } \\
\text { ion }\end{array}$ & $\begin{array}{l}\text { Miner } \\
\text { localization }\end{array}$ & $\begin{array}{l}\text { The main use of the coal mine } \\
\text { positioning system is to track and } \\
\text { manage the activities of the } \\
\text { underground workers. When the } \\
\text { accident occurred, the location } \\
\text { information by the miners, rescue } \\
\text { workers can quickly determine } \\
\text { the specific location of } \\
\text { underground personnel and } \\
\text { related conditions }\end{array}$ & $\begin{array}{l}<5 \\
\text { meters }\end{array}$ \\
\hline Traffic & $\begin{array}{l}\text { Satellite } \\
\text { navigation } \\
\text { aids }\end{array}$ & $\begin{array}{l}\text { The existing vehicle or pedestrian } \\
\text { navigation system (such as GPS/) } \\
\text { in the city center due to poor } \\
\text { satellite visibility, often can not } \\
\text { work effectively. You can achieve } \\
\text { seamless precision navigation } \\
\text { under various environments by } \\
\text { mixing Compass / WLAN } \\
\text { positioning system. }\end{array}$ & $\begin{array}{l}<5 \\
\text { meters }\end{array}$ \\
\hline $\begin{array}{l}\text { Industri } \\
\text { al } \\
\text { product } \\
\text { ion }\end{array}$ & $\begin{array}{l}\text { Parts } \\
\text { management } \\
; \\
\text { Production } \\
\text { Line } \\
\text { Monitoring }\end{array}$ & $\begin{array}{l}\text { By positioning the WLAN to } \\
\text { realize automatic management of } \\
\text { production parts, production tools } \\
\text { and production processes, to } \\
\text { increase productivity and shorten } \\
\text { the production cycle. }\end{array}$ & $\begin{array}{l}<1 \\
\text { meter }\end{array}$ \\
\hline $\begin{array}{l}\text { Logistic } \\
\text { s } \\
\text { manage } \\
\text { ment. }\end{array}$ & $\begin{array}{l}\text { Accurate } \\
\text { material } \\
\text { tracking and } \\
\text { warehouse } \\
\text { management }\end{array}$ & $\begin{array}{l}\text { The automatic recording and } \\
\text { tracking of the material location is } \\
\text { realized by WLAN, which can } \\
\text { realize the automation of } \\
\text { warehouse management. } \\
\text { According to the size of the } \\
\text { tracking of the material on the } \\
\text { positioning accuracy requirements } \\
\text { are also different. }\end{array}$ & $\begin{array}{l}<1 \\
\text { meters }\end{array}$ \\
\hline $\begin{array}{l}\text { Internet } \\
\text { or } \\
\text { mobile } \\
\text { Internet }\end{array}$ & $\begin{array}{l}\text { Location- } \\
\text { based } \\
\text { services } \\
\text { (LBS) and } \\
\text { precision } \\
\text { marketing }\end{array}$ & $\begin{array}{l}\text { The position and trajectory of the } \\
\text { user to enter the mall is obtained } \\
\text { by WLAN. Use this information, } \\
\text { accurate marketing system can } \\
\text { speculate the consumer behavior } \\
\text { and interest, and accordingly } \\
\text { targeted advertising push, } \\
\text { accurate to buy, discount, new } \\
\text { listing and other information } \\
\text { targeted to push to the user. }\end{array}$ & $\begin{array}{l}<5 \\
\text { meters }\end{array}$ \\
\hline $\begin{array}{l}\text { Govern } \\
\text { ment }\end{array}$ & $\begin{array}{l}\text { Crowd flow } \\
\text { management }\end{array}$ & $\begin{array}{l}\text { Through outdoor AP crossing the } \\
\text { smartphone passive positioning, to } \\
\text { achieve the position of the crowd, } \\
\text { the monitoring of the flow route, and } \\
\text { the real-time management of the } \\
\text { distribution of people. }\end{array}$ & $\begin{array}{l}<5 \\
\text { meters }\end{array}$ \\
\hline
\end{tabular}




\section{Conclusion}

With the rapid development of the internet of things related industries, various sectors in the field of business need more and more requirements on wireless location, especially the need to WLAN indoor positioning precision LBS location services. Based on TDOA and PTP high - precision WLAN positioning system, built on a software radio network communications platform, complete WiFi wireless signal arrival time accurate measurement, based on the principle of realization of the arrival time difference TDOA ranging positioning calculation; and innovation proposed based IEEE1588v2 (PTP) standards, protection of WLAN access points (AP) between the nanosecond level time synchronization accuracy; and finally, the system through NLOS error filtering and positioning before the data preprocessing, eliminate the influence of NLOS error, the time domain error control in wireless ranging $5 \mathrm{~ns}$ hereinafter, in order to achieve higher accuracy decimeter or location estimate.

The case can be widely used in mobile Internet, logistics management, mineral exploration, health and other needs of the precise location services (LBS) in other industry sectors. The system will be integrated into a multi-hop delay compensation, compensation for asymmetric algorithms such integrated application, highspeed positioning solver engine optimization, positioning and other open GIS Engine API interface, and gradually improve the system positioning accuracy,system integrators and end users to meet dimensional positioning business needs.

\section{References}

1. CCID Consulting, China location services (GPS / LBS) Market Competition Analysis Report [R / OL] [2014-07-14] . http //www.ccidconsulting.com/scyx/

2. Xu Jingwei. LBS system based on WLAN wireless location technology [D]. Wuhan: Huazhong University of Science and Technology master's degree thesis, 2011: 22-24

3. $\mathrm{Wu} Z$, et a1. Location Estimation via Support Vector Regression [J]. IEEE Transactions on Mobile Computing, 2012,6 (3)

4. Yan Xiaotian, warm bright students, Wuwei. WLAN positioning technology [EB / OL]. http://labs.chinamobile.com/mblog/712208_82775. 2012,3

5. Anthony LaMarca and Eyal de Lara Location Systems An Introduction to the Technology Behind Location Awarenes [J]. Morgan and Claypool, 2008,8 (3): $25-28$

6. MC Su, DY Huang, and SC Lin A regression-modebased approach to indoor location estimation [J] Journal of Engineering and Applied Sciences, 2008,3 (4):307-311

7. Huang Yi, Hu. Based on TDOA measurements of high-precision positioning technology research.
Aiqun high-tech communications, Vol. 22, No. 1.2012: 8-13

8. IEEE.IEEE Standard for a Precision Clock Synchronization Protocol for Networked Measurement and Control Systems [S] .IEEE1588v2, March 2008: 125-128.

9. Zhuoqun $\mathrm{Li}$, et al Range-based Relative Velocity Estimators for Networked Mobile Devices [J] .IEEE Transactions on Vehicular Technology, vol.58, issue 4, pp 2095 -2099, May 2009

10. Jingang Huang, Junjie Zhou. Software Process Improvement Based On CMM [C] .2013 AsiaPacific Computational Intelligence and Information Technology Conference .PP594-598.Dec 2013

11. Study of things for clock synchronization architecture $[\mathrm{J}]$ Bo and other Computer Engineering and Design 2011,32 (5): 16-21

12. Wang Yuan, Lin Jinguo. Discuss several common indoor positioning technology [J]. Chinese instruments 2011,2 (5): 23-26 